\title{
Guide to Authors: calling all authors!
}

\section{The 'Guide to Authors' provides detailed information on manuscript preparation and submission, the editorial process, and editorial and publishing policies. Awareness of these requirements can help avoid delays in the review and publication process.}

Nature Cell Biology's 'Guide to Authors' is updated at regular intervals as our editorial and publishing policies evolve in response to a dynamic publishing landscape and the changing needs of the research community. Crucial information on issues such as author responsibilities, availability of materials and data sharing, plagiarism and duplicate publication, can also be found at the 'Author and Referees' site. All authors are strongly encouraged to consult the 'Guide to Authors' before submission. Here, we highlight a few important points.

Submission policies. Manuscripts should be submitted through our online submission system, which allows authors to check the integrity of their uploaded data and monitor the status of their manuscript during the editorial and review process. The corresponding author is ultimately responsible for providing all relevant information and for communicating with the editors. Although it is the primary responsibility of the senior author(s) involved in the study to ensure the integrity of the data, submission is taken to imply that all the authors stand by the contents of the study. During the initial submission, or while a manuscript is under consideration at Nature Cell Biology, if related work from the same authors is submitted or accepted elsewhere they must provide us with a copy of that manuscript. In each case, the existence of related work should be mentioned in the cover letter. Conceptual advance is an important criterion at Nature Cell Biology, and the publication of related work (with or without overlapping authors) could jeopardize the success of a manuscript.

The editorial process and manuscript decisions. Nature journals do not have an advisory board of academic editors. The selection of manuscripts for formal review, and administration of the peer-review process, is managed solely by professional editors. The 'Guide to Authors' provides detailed information on the decision process for new submissions, revisions and appeals. Following submission, the handling editor evaluates the strengths of the study relative to the published literature, and then discusses the manuscript within the team of editors. Critical criteria include the degree of new insights, mechanistic elaboration and interest to a wide cell-biology readership. Nature Cell Biology can publish only a small fraction of the papers we receive; thus, rejection of manuscripts without peer review should not be viewed as a reflection on the technical quality of the work, or its significance to specialists.

Journal independence and manuscript transfer. Although Nature journals share editorial and publishing policies, they are editorially independent. Editors cannot influence the outcome of a decision at a different title, and a manuscript cannot be considered simultaneously at multiple Nature journals. However, authors may choose to transfer manuscripts between Nature journals, either following an editorial rejection or after the manuscript has been reviewed; a link at the bottom of the decision letter allows authors to exercise this option. In the case of manuscripts that have undergone review, the identities of the referees and their comments are also transferred and are taken into account when making a decision. Editors may also recommend a transfer to a sister journal; authors of transferred manuscripts should note that the editors at the second journal will make an independent decision about the suitability of the manuscript.

Plagiarism and image integrity. Nature Publishing Group is a member of the CrossCheck initiative, which allows editors to screen all submitted content against the CrossCheck database of published literature. Although we have yet to come across cases where authors have extensively duplicated text or data, we have occasionally encountered instances where sections of Methods have been copied verbatim from the author's previous publications. In such cases, authors will be asked to either rewrite the text, or remove it and reference the relevant publication.

In the age of Photoshop, it is all too easy to subject images to a level of processing that would be deemed unacceptable by our editorial standards. Authors are strongly encouraged to refer to the details of our guidelines for image integrity. To promote transparency in data presentation, we require authors to provide key gel and blot data in unprocessed form in the Supplementary Information. This journal also implements a policy of spot-checking two papers per issue that are either randomly selected or identified as potentially high-risk by editors or referees. The images are analysed by our production team and if evidence of unacceptable image manipulation is discovered, authors are asked to provide the original, raw data. In rare cases, authors might be asked to provide independent experimental evidence.

Statistical guidelines. Nature journals' guidelines on statistical analysis outline the information that must accompany quantitative data. At a minimum, quantitative data should include the precise number of independent experiments $(n)$ underlying each data point, an indication of the statistical significance, and definition of error bars. Details of statistical tests should be provided in the Methods. We also recommend that when $n$ is below 5, individual data points are plotted alongside an error bar.

Finally, production bound! Once a manuscript is accepted for publication in principle, the handling editor and the production team assess the text and figures for adherence to the editorial and production requirements. Our decision letter and the 'Guide to Authors' provide detailed information on preparing the manuscript text and figures. Strict adherence to these requirements can facilitate rapid publication. Common errors include failure to follow the guidelines for statistical analysis, providing images below the required resolution, and providing flattened multilayered images. If in doubt, authors are advised to contact the handling editor.

The editorial and production teams at Nature Cell Biology are committed to bringing thought-provoking research to our wide readership, and to facilitating a rapid and fair publication process. Author familiarity with the 'Guide to Authors' would help us fulfil these aims. 